\title{
APPLICATION OF MULTI-OBJECTIVE GENETIC ALGORITHM TO BRIDGE MAINTENANCE
}

\author{
H. Furuta, ${ }^{1}$ and T. Kameda ${ }^{2}$ \\ ${ }^{1}$ Department of Informatics, Kansai University, 2-1-1 Ryozenji-cho, Takatsuki, Osaka 569-1095, \\ Japan, furuta@res.kutc.kansai-u.ac.jp ${ }^{2}$ Graduate School of Informatics, Kansai University, 2- \\ 1-1 Ryozenji-cho, Takatsuki, Osaka 569-1095, Japan
}

\begin{abstract}
In order to establish a rational bridge management program, it is necessary to develop a cost-effective decision-support system for the maintenance of bridges. In this paper, an attempt is made to develop a new multi-objective genetic algorithm for the bridge management system that can provide practical maintenance plans. Several numerical examples are presented to demonstrate the applicability and efficiency of the proposed method.
\end{abstract}

keywords: Bridge Maintenance, Genetic Algorithm, Life-Cycle Cost, MultiObjective Optimization, Repair

\section{INTRODUCTION}

In this paper, an attempt is made to apply a Multi-Objective Genetic Algorithm (MOGA) for the establishment of optimal planning of existing bridge structures. In order to establish a rational maintenance program for the bridge structures, it is necessary to evaluate the structural performance of existing bridges in a quantitative manner. So far, several structural performance indices have been developed, some of which are reliability, durability, and damage indices. However, it is often necessary to discuss the structural performance from the economic and/or social points of view.

Life-Cycle Cost (LCC) is one of useful measures for evaluating the structural performance from another standpoint. Using these measures, the overall cost can be reduced and an appropriate allocation of resources can be achieved. In general, LCC optimization is to minimize the expected total cost which includes the initial cost involving design and construction, routine or preventive maintenance cost, inspection, repair and failure costs. Then, the optimal strategy obtained by LCC optimization can be different according to the prescribed level of structural performance and required service life. 


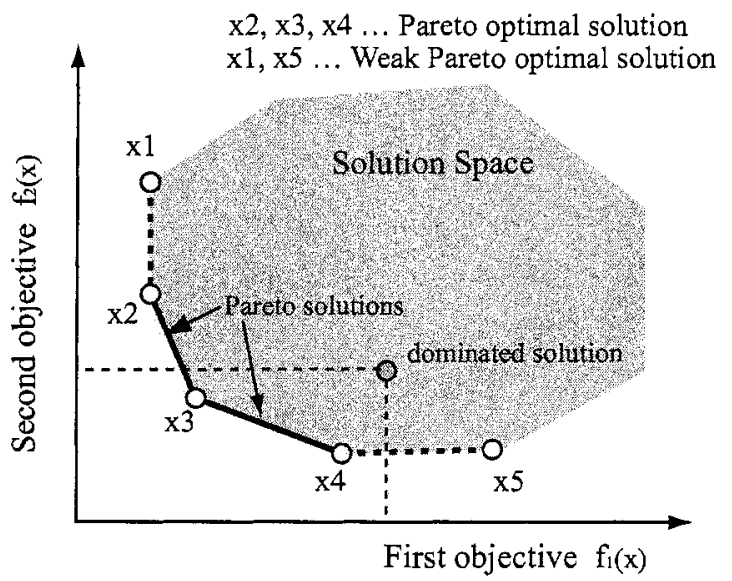

Figure 1. Pareto solutions

In this paper, an attempt is made to discuss the relationships among several performance measures and provide rational balances of these measures by using MOGA. Furthermore, another attempt is made to develop a new multiobjective genetic algorithm for the bridge management problem that have a lot of constraints. Several numerical examples are presented to demonstrate the applicability and efficiency of the proposed method.

\section{MULTI-OBJECTIVE GENETIC ALGORITHM}

\subsection{Multi-Objective Problem}

A multiple-objective optimization problem has two or more objective functions that cannot be integrated into a single objective function [1]. In general, the objective functions cannot be simultaneously minimized (or maximized). It is the essence of the problem that trade-off relations exist among the objective functions. The concept of "Pareto optimum" becomes important in order to balance the trade-off relations. The Pareto optimum solution is a solution that cannot improve an objective function without sacrificing other functions (Figures 1 and 2). A dominated, also called non-dominant, solution is indicated in Figure 1.

\subsection{Multi-Objective Genetic Algorithm (MOGA)}

Genetic Algorithm (GA) is an evolutionary computing technique, in which candidates of solutions are mapped into GA space by encoding. The following steps are employed to obtain the optimal solutions [2]: a) initialization, b) 


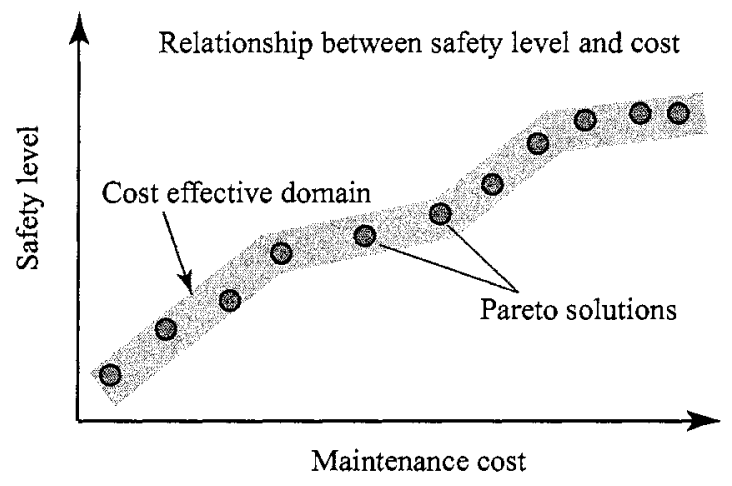

Figure 2. Cost-effective domain including Pareto solutions

crossover, c) mutation, d) natural selection, and e) reproduction. Individuals, which are solution candidates, are initially generated at random. Then, steps $\mathrm{b}, \mathrm{c}, \mathrm{d}$, and e are repeatedly implemented until the termination condition is fulfilled. Each individual has a fitness value to the environment. The environment corresponds to the problem space and the fitness value corresponds to the evaluation value of objective function. Each individual has two aspects: Gene Type (GTYPE) expressing the chromosome or DNA and Phenomenon Type (PTYPE) expressing the solution. GA operations are applied to GTYPE and generate new children from parents (individuals) by effective searches in the problem space, and extend the search space by mutation to enhance the possibility of individuals other than the neighbor of the solution. GA operations that generate useful children from their parents are performed by crossover operation of chromosome or genes (GTYPE) without using special knowledge and intelligence. This characteristic is considered as one of the reasons of the successful applications of GA [3].

\section{FORMULATION OF BRIDGE MAINTENANCE PLANNING}

It is desirable to determine an appropriate life-cycle maintenance plan by comparing several solutions for various conditions [4][5]. A new decision support system is developed here from the viewpoint of multi-objective optimization, in order to provide various solutions needed for the decision-making.

In this study, LCC, safety level and service life are used as objective functions. LCC is minimized, safety level is maximized, and service life is maximized. There are trade-off relations among the three objective functions. For example, LCC increases when service life is extended, and safety level and service life 


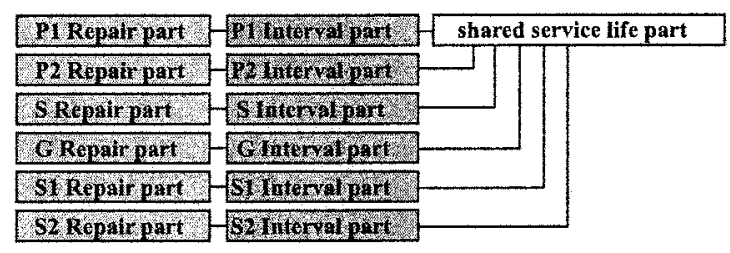

P1 : upper pier, P2 : lower pier, S : shoe, G : girder, S1 : slab (central section), $\mathrm{S} 2$ : slab (bearing section)

Figure 3. Structure of DNA

GTYPE

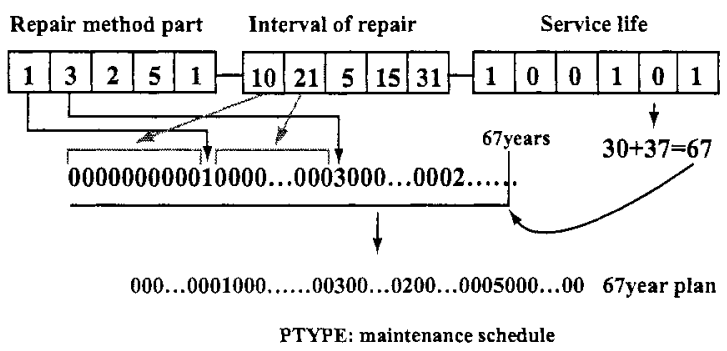

Figure 4. Coding rule

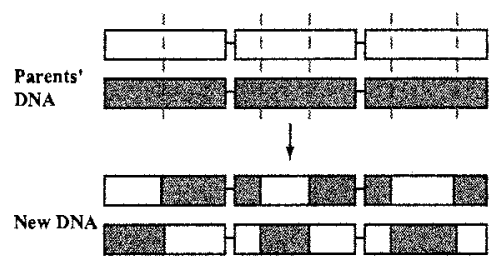

Figure 5. Crossover

decrease due to the reduction of LCC. Then, multi-objective optimization can provide a set of Pareto solutions that can not improve an objective function without making other objective functions worse [6].

In the proposed system, DNA structure is constituted as shown in Figure 3, in which DNA of each individual consists of three parts such as repair method, interval of repair, and shared service life (Figure 4). In this figure, service life is calculated as the sum of repairing years and their interval years. In Figure 4, service life is obtained as 67 years which is expressed as the sum of 30 years and 37 years. The repair part and the interval part have the same length. Gene 


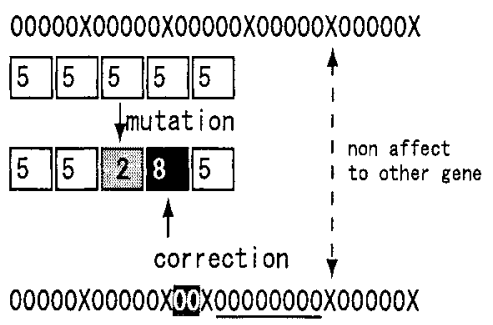

Figure 6. Mutation

of repair part has ID number of repair method. The interval part has enough length to consider service life. In this system, ID 1 means surface painting, ID 2 surface coating, ID 3 section restoring, ID 4 desalting (re-alkalization) or cathodic protection, and ID 5 section restoring with surface covering. DNA of service life part has a binary expression with six bits and its value is changed to decimal number.

In crossover, the system generates new candidates by using the procedure shown in Figure 5. For mutation, the system shown in Figure 6 is used.

Then, objective functions are defined as follows:

$$
\begin{gathered}
\text { Objectivefunction } 1: C_{\text {total }}=\sum L C C_{i} \rightarrow \text { min } \\
\text { where } L C C_{i}=\mathrm{LCC} \text { for bridge } \\
\text { Objective function } 2: Y_{\text {total }}=\sum Y_{i} \rightarrow \max \\
\text { Constraints : } Y_{i}>Y_{\text {required }} \\
\text { where } Y_{i}=\text { Service life of bridge } Y_{i}, Y_{\text {required }}=\text { Required service life }
\end{gathered}
$$




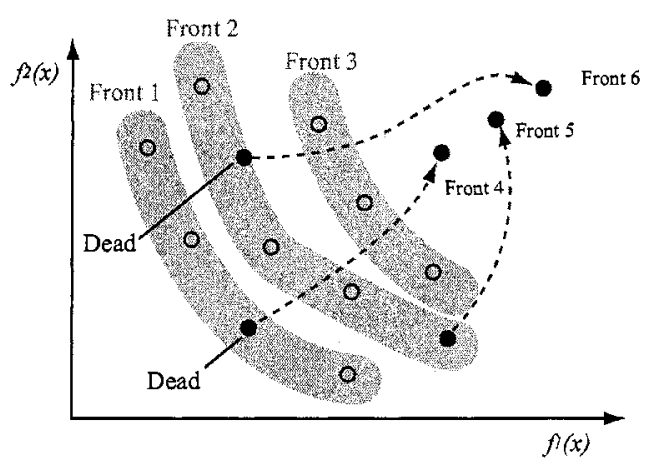

Figure 7. Concept of Front

\section{NEW MULTI-OBJECTIVE GENETIC ALGORITHM}

As mentioned before, the formulation of bridge maintenance planning has several constraint conditions. In usual, it is not easy to solve multi-objective optimization problems with constraints by applying the multi-objective genetic algorithm.

In this study, an improvement is made on the selection process by introducing the sorting technique. The selection is performed using so-called sorting rules which arrange the list of individuals in the order of higher evaluation values. Then, the fitness values are assigned to them by using the linear normalization technique. In usual, if the fitness values are calculated directly according to the evaluation values, the differences among every individuals decrease so that the effective selection can not be done. On the other hand, the linear normalization technique enables to keep the selection pressure constant so that it may continue the selection well. In this study, the selection procedure is improved coupling the linear normalization technique and the sorting technique. Using the evaluation values, the individuals are reordered and given the new fitness values. Figure 7 presents the process of the selection proposed here. The individuals of satisfying the constraints are arranged first according to the evaluation values and followingly the individuals of unsatisfying the constraints are arranged according to the degree of violating the constraints. Accordingly, all the individuals are given the fitness values using the linear normalization technique.

In order to apply the sorting rules to the multi-objective optimization problems, the non-dominated sorting method is used. In the non-dominated sorting method, the Pareto solutions are defined as Front1. Then, Front 2 is determined by eliminating the Front 1 from the set of solution candidates. Repeating the process, the new Front is pursued until the solution candidates diminish. Fur- 
ther, the Fronts are stored in the pool of the next generation. If the pool is full, the individuals in the Front are divided into the solutions to survive or die based on the degree of congestion.

In this study, the individuals are divided into the group of satisfying the constraints and the group without satisfying the constraints. The former is called as live individual, and the latter dead individual. While the live individuals are given the fitness values according to the evaluation values after the nondominated sorting, the dead individuals are given the same fitness value. When implementing the non-dominated sorting, the Pareto front may not exist at the initial generation, because there remain a lot of dead individuals after the nondominated sorting. Then, the dead individuals are arranged in the order of degree of violating the constraints and some of them are selected for the next generation. Thus, the multi-objective optimization problem with constraints are transformed into the minimization problem of violation of constraints. The elite preserve strategy is employed for the selection of survival individuals.

When the generation is progressed, live individuals appear and then both the live individuals forming the Pareto front and the dead individuals arranged in the order of violation degree exist together. In this case, appropriate numbers of live and dead individuals are selected for the next generation. If the number of individuals involved in the Pareto front increases, only the individuals are selected for the next generation.

\section{NUMERICAL EXAMPLES}

Figures 5 through 9 present the results calculated for B01 model (Bridge 1) with the constraint that safety level should be greater than 0.6 under the environment that neutralization is dominant. From Figure 5, it is seen that the number of live individuals immediately increases after 20 generations and reaches to 1000 at 40 generations. Similarly, the number of Pareto solutions increases with some fluctuation and converges after 125 generations. As the generation proceeds from 100 to 3000 , the Pareto solutions can be improved smoothly. Figure 5 shows that the proposed method can provide solutions satisfying the safety requirement very well. Figure 10 shows the repair methods given by the proposed method. Figure 11 presents the results of B04, which gives the safety for every structural element that is greater than the prescribed target value 0.6 , though the safety levels are different. Figure 12 shows the optimal combination of repair methods.

\section{CONCLUSIONS}

In this paper, an attempt was made to formulate the optimal maintenance planning as a multi-objective optimization problem. By considering LCC, safety level, and service life as objective functions, it is possible to obtain 


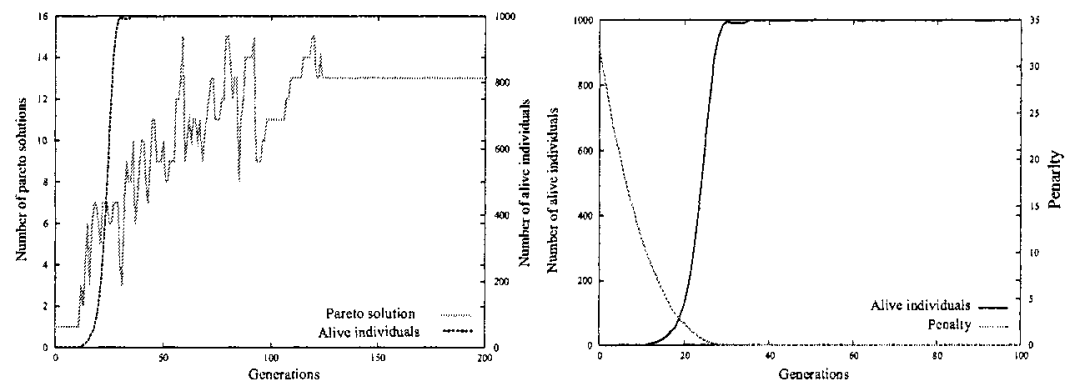

Figure 8. Calculated result for bridge 1 under the neutralization environment -relation between number of Pareto solutions and generation (left) and live solutions and generation (right)

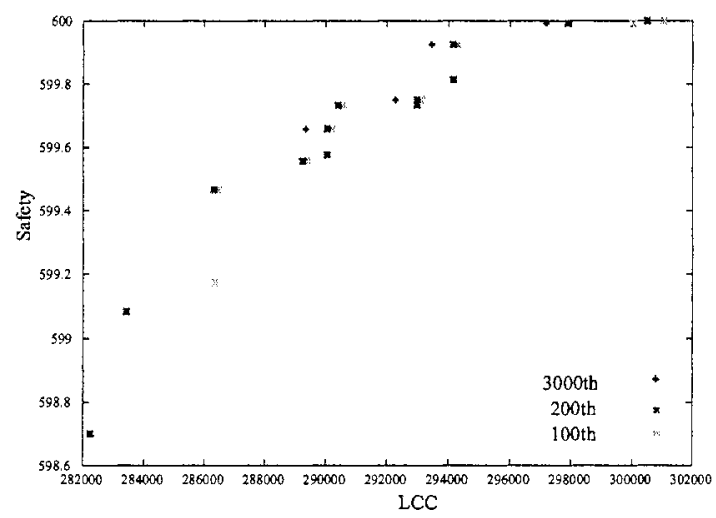

Figure 9. Calculated result for bridge 1 under the neutralization environment-relation between safety level and LCC-

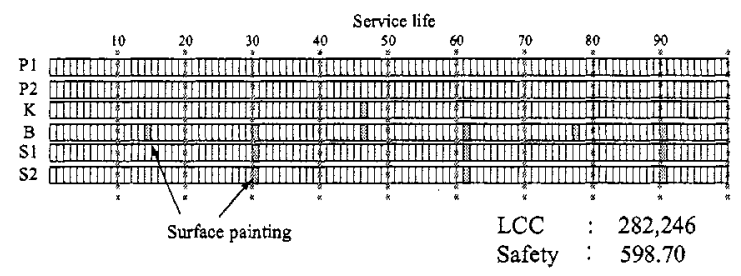

Figure 10. Repair methods for bridge 1 


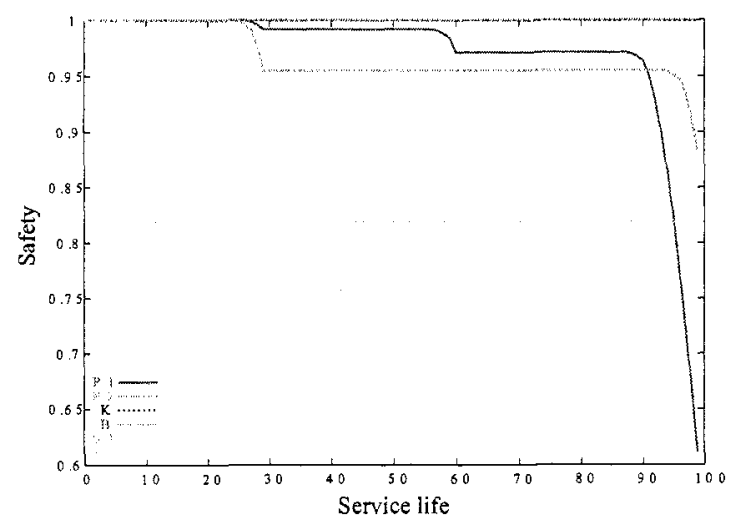

Figure 11. Change of safety level of bridge 4

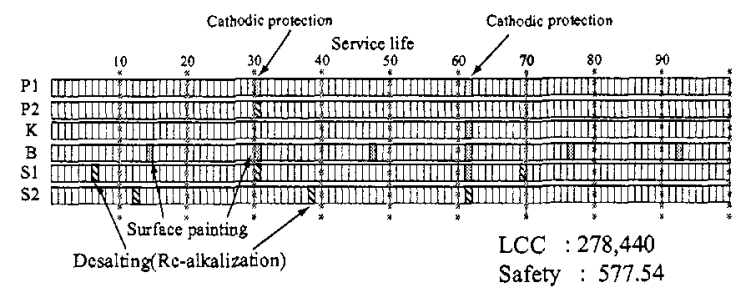

Figure 12. Repair methods for bridge 4 
the relationships among these three performance indicators and provide bridge maintenance engineers with various maintenance plans with appropriate allocations of resources. Furthermore, another attempt was made to develop a new multi-objective genetic algorithm for the bridge management problem that has a lot of constraints. Based on the results presented in this paper, the following conclusions may be drawn:

- Since the optimal maintenance problem is a very complex combinatorial problem, it is difficult to obtain reasonable solutions by the current optimization techniques.

- Although Genetic Algorithm (GA) is applicable to solve multi-objective problems, it is difficult to apply it to large and very complex bridge maintenance problems. By introducing the technique of Non-Dominated Sorting GA-2 (NSGA2), it is possible to obtain efficient near-optimal solutions for the maintenance planning of a group of bridge structures.

- Furthermore, the new GA method proposed here can much more improve the convergence and efficiency in the optimization procedure, by introducing the sorting based selection.

- The proposed method can provide many near-optimal maintenance plans with various reasonable LCC values, safety levels and service lives.

\section{References}

[1] Iri, M. Konno, H. (eds.).Multi-Objective Optimization, Tokyo, Sangyo-tosho(in Japanese). 1982

[2] Goldberg, D.E. Genetic Algorithms in Search, Optimization and Machine Learning, Addison Wesley Publishing, 1989.

[3] Furuta, H. Sugimoto, H. Applications of Genetic Algorithm to Structural Engineering, Tokyo, Morikita Publishing (in Japanese). 1997

[4] Furuta, H., Kameda, T., Fukuda, Y., and Frangopol, D. M. Life-Cycle Cost Analysis for Infrastructure Systems: Life Cycle Cost vs. Safety Level vs. Service Life, Proceedings of Joint International Workshops LCCO3/IABMAS and fip/JCSS, EPFL, Lausanne, March 24-26,2003,

(keynote lecture); in Life-Cycle Performance of Deteriorating Structures: Assessment, Design and Management, Frangopol, D. M., Bruhwiler, E., Faber, M. H. and Adey, B. (eds.), ASCE, Reston, Virginia, 19-25.

[5] Furuta, H., Kameda, T. and Frangopol, D. M. Balance of Structural Performance Measures, Proc. of Structures Congress, Nashville, Tennessee, ASCE, May, CD-ROM. 2004

[6] Liu, M. and Frangopol, D.M. (2004b). Optimal Bridge Maintenance Planning Based on Probabilistic Performance Prediction, Engineering Structures, Elsevier, 26(7), 991-1002. 